\title{
Comparison of Fetomaternal Outcomes in Patients With Gestational Diabetes Mellitus Treated With Insulin Versus Acarbose: Results of a Prospective, Open Label, Controlled Study
}

Suryakanta Jayasingh Sr. ${ }^{1}$, Saumya Nanda ${ }^{2}$, Sujata Misra ${ }^{3}$, A Baliarsinha ${ }^{4}$, Sidhartha Das ${ }^{5}$, Anant Patil ${ }^{6}$

1. Obstetrics and Gynecology, Institute of Medical Sciences and SUM Hospital, Bhubaneswar, IND 2. Obstetrics and Gynecology, Srirama Chandra Bhanja Medical College, Cuttack, IND 3. Obstetrics and Gynecology, Fakir Mohan Medical College, Balasore, IND 4. Endocrinology, Srirama Chandra Bhanja Medical College, Cuttack, IND 5. Internal Medicine, Srirama Chandra Bhanja Medical College, Cuttack, IND 6. Pharmacology, Dnyandeo Yashwantrao Patil University-School of Medicine, Navi Mumbai, IND

Corresponding author: Saumya Nanda, saumyananda81@gmail.com

\section{Abstract \\ Objective}

To evaluate fetomaternal outcomes in patients with gestational diabetes mellitus (GDM) treated with insulin versus acarbose.

\section{Material and methods}

In this prospective, open label, controlled study, GDM patients treated with insulin or acarbose were observed till six weeks after delivery. Maternal outcomes, fetal outcomes and glycemic control were compared between two groups.

\section{Results}

Fifty patients in each group (insulin group-mean age 28.52 years; acarbose group-mean age 26.26 years; $\mathrm{p}=0.020$ ) were included. There was no difference in body mass index $(\mathrm{p}=0.157)$, family history of diabetes $(p=0.648)$, history of GDM $(p=0.50)$ or mean gestational age at diagnosis $(p=0.245)$ between the two groups. There was no significant difference in the incidence of recurrent infections $(\mathrm{p}=0.64)$, pre-eclampsia $(\mathrm{p}=0.64)$ or premature rupture of membranes $(\mathrm{p}=0.40)$ between the two groups. Mean duration of gestational weeks at the time of delivery in the insulin and acarbose group was 36.93 and 38.36 weeks respectively $(p=0.002)$. There was no difference in the modes of delivery, mean post-operative random blood glucose $(\mathrm{p}=0.96)$, fasting blood glucose level at day seven $(\mathrm{p}=0.15)$ and after six weeks $(\mathrm{p}=0.83)$ between the insulin and acarbose groups. There was no difference in reduction in the postprandial blood glucose level at day seven $(p=0.48)$ and after six weeks $(p=0.23)$. There was no significant difference in the mean birth weight of babies born to mothers treated with the two drugs $(\mathrm{p}=0.21)$. There was no difference in the incidence of neonatal complications between the two groups.

Review began 12/08/2020 Review ended 12/15/2020 Published 12/25/2020

\section{() Copyright 2020}

Jayasingh et al. This is an open access article distributed under the terms of the Creative Commons Attribution License CC-BY 4.0., which permits unrestricted use, distribution, and reproduction in any medium, provided the original author and source are credited.

\section{Conclusion}

Acarbose can be an effective and well tolerated option for treatment of gestational diabetes mellitus.

Categories: Internal Medicine, Obstetrics/Gynecology

Keywords: acarbose, fetal outcomes, gestational diabetes, insulin

\section{Introduction}

Gestational diabetes mellitus (GDM) is a condition of glucose intolerance observed during pregnancy. It occurs due to defects in the response by the beta cells of pancreas to insulin during pregnancy and is one of the known common complications during pregnancy [1-4]. According to the results of a meta-analysis, overall prevalence of GDM in Europe is 5.4\% [5]. Overall prevalence of GDM in different countries ranges from less than $2 \%$ to $17 \%$. The rates differ between geographical areas and the type of population being studied. The literature also suggests rising rates of GDM [2]. Incidence of GDM is increasing worldwide [6].

Women with a history of GDM have a significantly higher risk of developing type 2 diabetes mellitus as compared to those without GDM $[1,7]$. Similarly, risk of metabolic syndrome is higher in patients with GDM than those without it [7]. Diagnosis and treatment of GDM is important to avoid maternal and fetal complications $[8,9]$.

Considering its pharmacological properties, insulin therapy is the first-line drug therapy for the treatment of 
GDM [8]. It is considered as the gold standard therapy in patients who are unable to control their glycemia with lifestyle measures [10]. Requirements of insulin differ from woman to woman, with some requiring lower doses while others may need significantly higher doses [8]. However, use of insulin is associated with several limitations including need for injections, pain at the site of injection and cost of treatment.

With this background, clinicians are always in search of an alternative option. Although several oral agents are available in the market, their usage in pregnancy is controversial. Metformin crosses the placenta, but its usage is not associated with significant concerns in newborns [4,10]. Some data is available on the use of glyburide and acarbose in gestational diabetes mellitus [11]. According to a meta-analysis, glyburide is inferior than metformin and insulin in GDM [12].

Acarbose is an alpha glucosidase inhibitor which reduces absorption of carbohydrates from the small intestine by inhibiting breakdown of disaccharides and oligosaccharides. This helps in reducing the risk of postprandial hyperglycemia. Considering minimal absorption in the circulation, acarbose seems to be a promising option for the treatment of gestational diabetes mellitus. Currently, the ACARB-GDM-a phase III prospective, multi-center, non-inferiority, randomized trial is on-going to study acarbose and prandial insulin as a treatment of GDM [13]. There is lack of data from well-designed clinical trials in gestational diabetes mellitus among patients from India.

The objective of this study was to evaluate fetomaternal outcomes in women with GDM treated with insulin versus acarbose.

\section{Materials And Methods}

In this prospective, open label and controlled study, pregnant women attending the obstetrics outpatient department between January 2017-January 2018 who underwent 75 gm oral glucose tolerance test and those having fasting plasma glucose more than $92 \mathrm{mg} / \mathrm{dl}$, one hour postprandial glucose level more than $180 \mathrm{mg} / \mathrm{dl}$ and two hour postprandial glucose level more than $153 \mathrm{mg} / \mathrm{dl}$ were included in the study. Patients with multifetal gestations, pre-gestational diabetes (type 1 or type 2 diabetes mellitus), diabetes diagnosed before 10 weeks of gestation or GDM patients with other chronic diseases and gestational age at delivery less than 20 weeks were excluded.

Patients treated with acarbose and insulin were observed for outcomes until six weeks after delivery. Maternal outcomes i.e. incidence of recurrent genitourinary infections, pre-eclampsia, premature rupture of membranes, gestational age at the time of delivery and modes of delivery were compared between two groups. Post-operative/delivery random blood glucose, fasting blood glucose and postprandial glucose at day seven and after six weeks was checked in mothers treated with both the drugs. Glycosylated haemoglobin was examined post six weeks after delivery in both groups. In new born babies, mean (SD) birth weight was calculated. Mean (SD) random blood glucose levels were checked in babies in the both groups. Incidence of babies with birth weight of more than $3500 \mathrm{~g}$, intrauterine growth retardation, birth trauma, birth asphyxia, neonatal jaundice, admission to the special new born care unit and congenital anomaly was calculated and compared between mothers with gestation diabetes treated with insulin versus acarbose.

Continuous data is presented as mean and standard deviation whereas categorical data is presented as number and percentages. Chi-square test and students unpaired ' $t$ ' test were used for comparison of categorical data and continuous data between two groups respectively. P value of less than 0.05 was considered significant.

\section{Results}

A total of 100 patients (acarbose group $n=50$ and insulin $n=50$ ) were included in the study. The mean age of the patients treated with insulin and acarbose was 28.52 years and 26.26 years respectively $(\mathrm{p}=0.020)$. There was no significant difference in body mass index of patients between two groups ( $\mathrm{p}=0.157$; Table 1 ) 


\section{Cureus}

\begin{tabular}{|c|c|c|c|}
\hline & Insulin $(n=50)$ & Acarbose $(n=50)$ & P value \\
\hline Mean (SD) age in years & $28.52(5.09)$ & $26.26(4.4)$ & 0.020 \\
\hline Mean (SD) BMI (kg/m²) & $26.50(3.20)$ & $25.56(3.28)$ & 0.157 \\
\hline Primigravida n (\%) & 14(28) & 18(36) & \multirow{2}{*}{0.39} \\
\hline Multigravida n (\%) & $36(72)$ & $32(64)$ & \\
\hline Primipara n (\%) & $20(40)$ & 27(54) & \multirow{2}{*}{0.16} \\
\hline Multipara n (\%) & $30(60)$ & 23(46) & \\
\hline Hypertension & $5(10 \%)$ & $9(18 \%)$ & 0.249 \\
\hline Thyroid disease & $6(12 \%)$ & $7(14 \%)$ & 0.766 \\
\hline Family history of diabetes $\mathrm{n}(\%)$ & $14(28 \%)$ & $12(24 \%)$ & 0.648 \\
\hline History of gestational diabetes mellitus $n(\%)$ & $16(32 \%)$ & $13(26 \%)$ & 0.50 \\
\hline Mean (SD) gestational age at the time of diagnosis in weeks & $16.86(11.71)$ & $16.08(2.94)$ & 0.245 \\
\hline Mean (SD) blood glucose after 2 hours of $75 \mathrm{gm}$ glucose tolerance test ( $\mathrm{mg} / \mathrm{dl}$ ) & 166.68 (11.71) & $163.72(6.35)$ & 0.119 \\
\hline Mean (SD) fasting blood glucose at the time of diagnosis (mg/dl) & $110.44(7.80)$ & $111.82(7.56)$ & 0.372 \\
\hline Mean (SD) postprandial blood glucose $(\mathrm{mg} / \mathrm{dl})$ at the time of diagnosis & $164.14(16.31)$ & 162.66 (16.31) & 0.560 \\
\hline
\end{tabular}

TABLE 1: Baseline characteristics of patients in both groups

There was no significant difference between the two groups in terms of past history of hypertension $(\mathrm{p}=0.249)$, thyroid disease $(\mathrm{p}=0.766)$, family history of diabetes $(\mathrm{p}=0.648)$ or history of gestational diabetes mellitus ( $\mathrm{p}=0.50)$. A total of $14(28 \%)$ and 18 (36\%) of patients in the insulin and acarbose groups respectively were primigravida $(\mathrm{p}=0.39)$, whereas $30(60 \%)$ and $23(46 \%)$ patients in the insulin and acarbose group were multiparous respectively $(\mathrm{p}=0.16)$. There was no significant difference in the mean gestational age at diagnosis between patients in the insulin group versus those in acarbose group (16.86 vs 16.08 weeks; $\mathrm{p}=0.245$ ). Similarly, there was no significant difference in the mean blood glucose after two hours of $75 \mathrm{~g}$ of glucose tolerance test $(\mathrm{p}=0.119)$, mean fasting blood glucose at diagnosis $(\mathrm{p}=0.372)$ and mean postprandial blood glucose $(\mathrm{p}=0.560)$ at the time of diagnosis.

There was no significant difference in the reduction of mean (SD) fasting blood glucose level at day seven [99.80 (15) vs 103.58 (10.78); $\mathrm{p}=0.15$ ) and after six weeks [99.84 (14.78) vs 99.30 (11.56); $\mathrm{p}=0.83$ ] between the insulin and acarbose groups respectively (Figure 1).

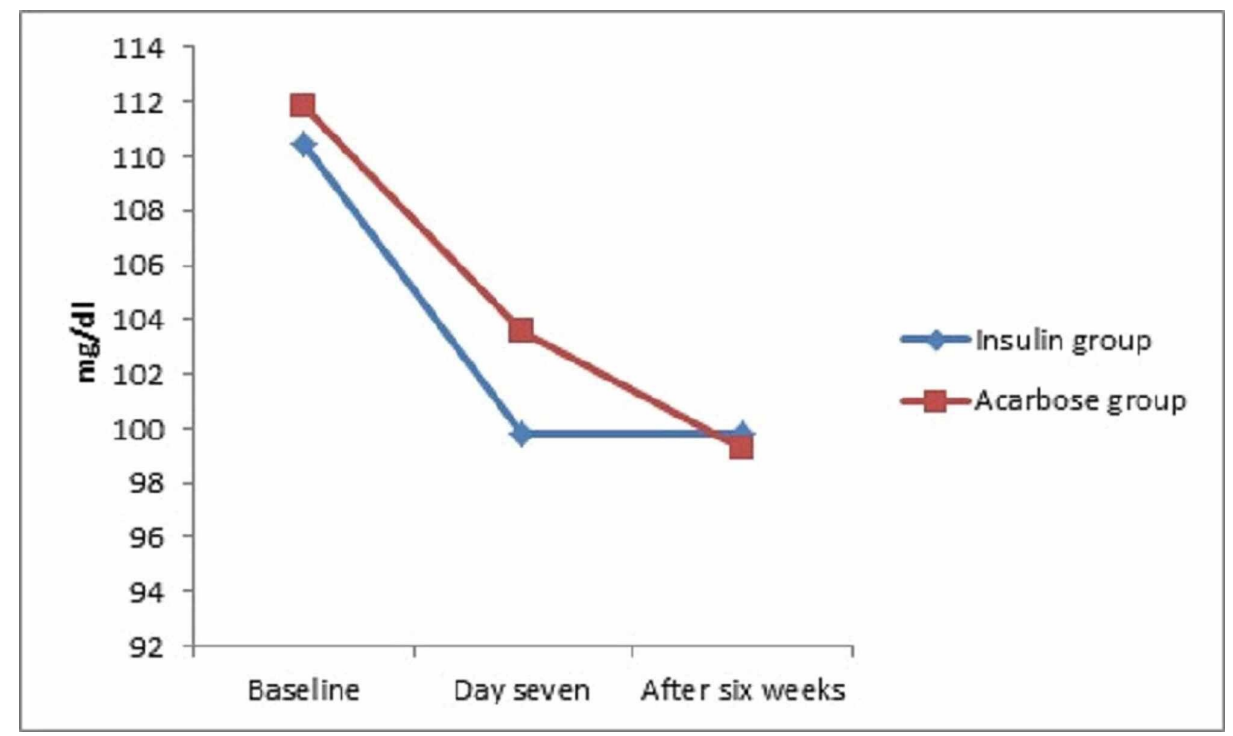

FIGURE 1: Reduction in mean fasting blood glucose in patients with 


\section{Cureus}

There was no significant difference in reduction in the mean (SD) postprandial blood glucose level at day seven [146.52 (32.12) vs 142.92 (16.38); $\mathrm{p}=0.48$ ) and after six weeks [141.44 (21.37) vs 137.08 (14.54); $\mathrm{p}=0.23$ ] between the insulin and acarbose groups respectively (Figure 2).

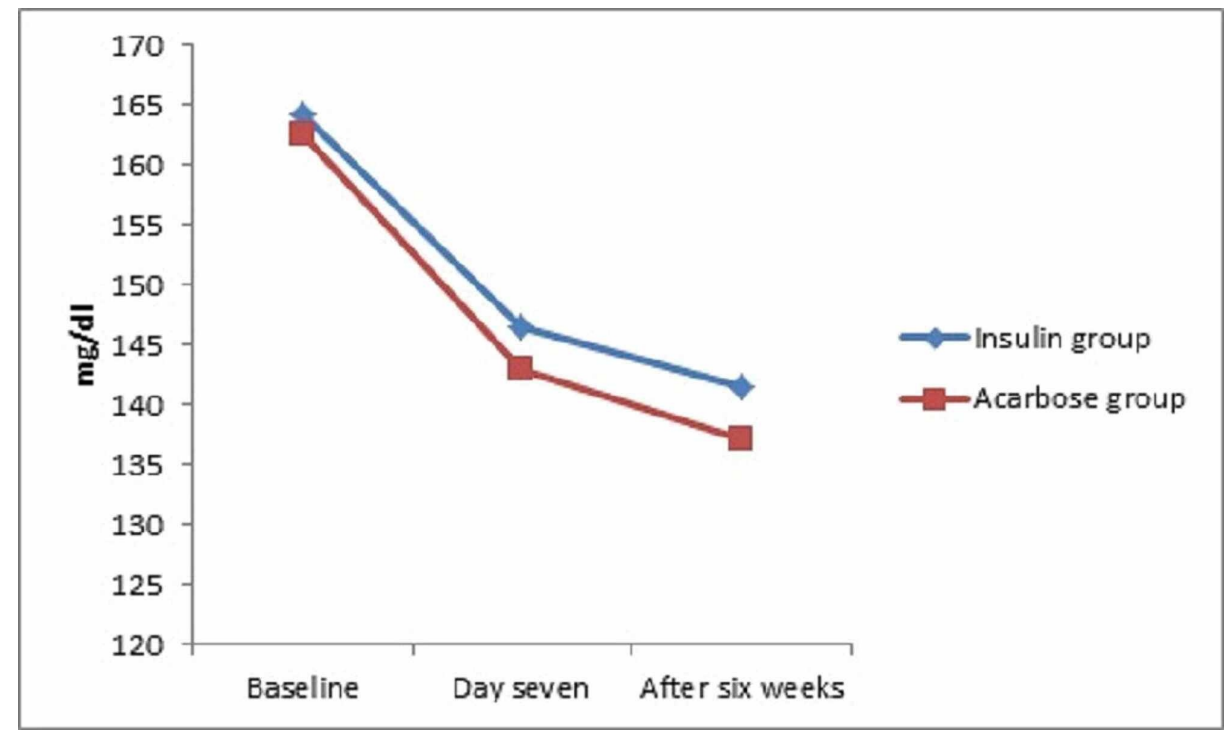

FIGURE 2: Reduction in mean post-prandial blood glucose in patients with gestational diabetes treated with insulin and acarbose

Similarly, mean (SD) HbA1c levels in the insulin group [6.50 (0.41)] and acarbose group [6.42 (0.30)] were similar ( $\mathrm{p}=0.82$ ). During the antenatal period, $10(20 \%)$ of the patients treated with insulin and $9(18 \%)$ of the patients treated with acarbose developed recurrent infections $(\mathrm{p}=0.64)$. A total of $14(28 \%)$ of the patients treated with insulin and $12(24 \%)$ of the patients treated with acarbose developed preeclampsia $(\mathrm{p}=0.64)$. Preterm-premature rupture of membranes (PPROM)/premature rupture of membranes (PROM) was seen in $16(32 \%)$ of the cases treated with insulin versus $20(40 \%)$ of the cases treated with acarbose $(\mathrm{p}=0.40)$ (Table 2).

\begin{tabular}{|c|c|c|c|}
\hline & $\begin{array}{l}\text { Insulin } \\
(n=50)\end{array}$ & $\begin{array}{l}\text { Acarbose } \\
(\mathrm{n}=50)\end{array}$ & $\begin{array}{l}P \\
\text { value }\end{array}$ \\
\hline Recurrent genitourinary infections & $10(20 \%)$ & $9(18 \%)$ & 0.79 \\
\hline Preeclampsia & $14(28 \%)$ & $12(24 \%)$ & 0.64 \\
\hline $\begin{array}{l}\text { Preterm-Premature rupture of membranes (PROM)/Premature rupture of membranes } \\
\text { (PROM) }\end{array}$ & $16(32 \%)$ & $20(40 \%)$ & 0.40 \\
\hline Delivery at mean (SD) gestational weeks & $36.93(2.71)$ & $38.36(1.68)$ & 0.002 \\
\hline Lower (uterine) segment caesarean section & $28(56 \%)$ & $33(66 \%)$ & 0.55 \\
\hline Vaginal delivery & $19(38 \%)$ & $14(28 \%)$ & 0.55 \\
\hline Instrumental delivery & $3(6 \%)$ & $3(6 \%)$ & 0.55 \\
\hline
\end{tabular}

TABLE 2: Maternal outcomes in two study groups

The mean (SD) duration of gestational week at the time of delivery in the insulin treated group and acarbose treated groups was 36.93 (2.71) and 38.36 (1.68) weeks respectively ( $\mathrm{p}=0.002)$. There was no significant difference in the modes of delivery between two groups for lower (uterine) segment caesarean section $(\mathrm{p}=0.55)$, vaginal delivery $(\mathrm{p}=0.55)$ and instrumental delivery $(\mathrm{p}=0.55)$. There was no difference in the mean 
(SD) post-operative random blood glucose in insulin treated group versus acarbose treated group [113.38 (25.85) vs 113.66 (29.48) $\mathrm{mg} / \mathrm{dl} ; \mathrm{p}=0.96]$.

There was no significant difference in the mean (SD) birth weight of babies born to mothers treated with insulin versus acarbose [2580 (720.02) vs 2744 (577.17) grams; p=0.21]. Similarly, there was no significant difference in the mean (SD) random blood glucose levels in babies born to mothers after insulin treatment versus those born to mothers treated with acarbose [58.92 (12.08) vs 57 (14.06) $\mathrm{mg} / \mathrm{dl})$; $\mathrm{p}=0.46$ ]. A total of 6 (12\%) babies in both groups had random blood glucose less than $45 \mathrm{mg} / \mathrm{dl}$ after delivery.

A total of 2(4\%) and 4 (8\%) babies in the insulin group and acarbose group had birth weight of more than $3500 \mathrm{~g}$ respectively. Intrauterine growth retardation was observed in 9 (18\%) and 7 (14\%) babies in the insulin and acarbose group respectively.

There was no difference in the incidence of birth trauma [3 (6\%) vs $6(12 \%)$; $=0.29$ ], shoulder dystocia [ 7 (14\%) vs 5 (10\%); p=0.53], birth asphyxia [17 (34\%) vs 18 (36\%); p=0.83], neonatal jaundice [22 (44\%) vs 21 (42\%); $\mathrm{p}=0.84$ ], admission to special new born care unit [27 (54\%) vs $30(60 \%), \mathrm{p}=0.54$ ] and congenital anomaly [ 1 (2\%) vs $0 \%$; $=0.31$ ] between patients treated with insulin versus acarbose respectively (Figure 3).

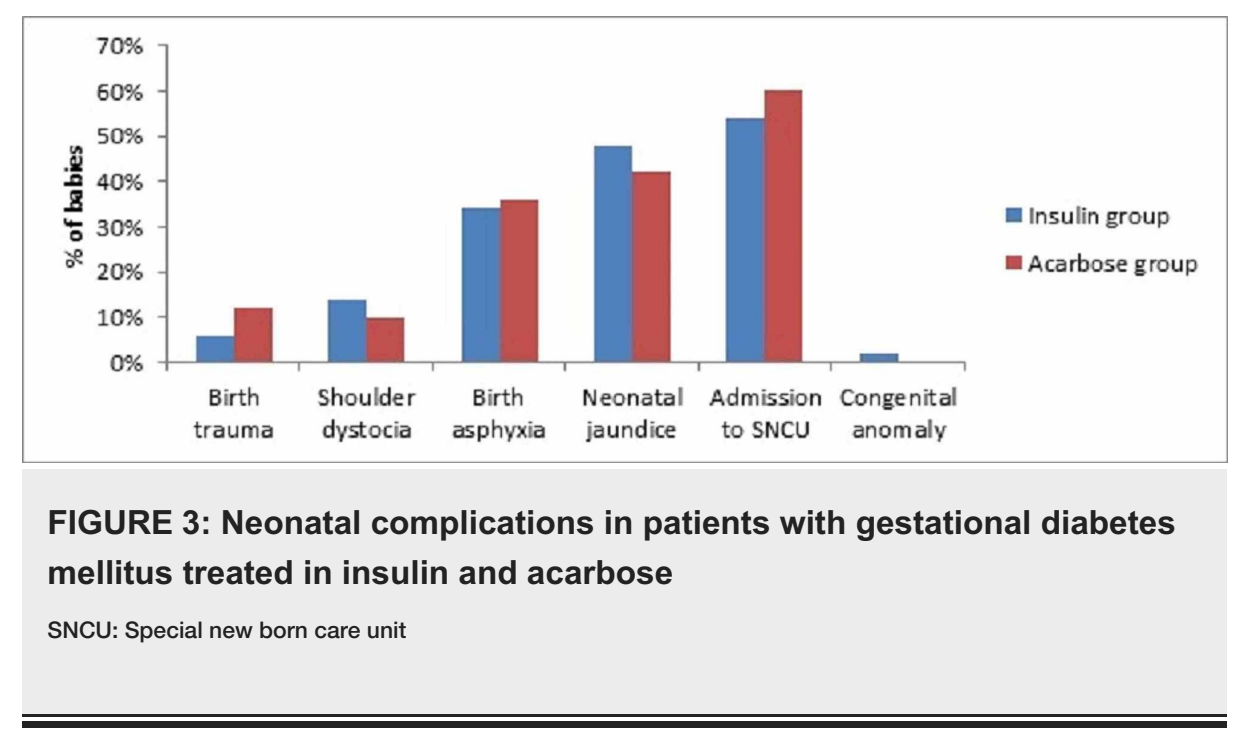

\section{Discussion}

It is important to recognize GDM and treat it effectively to avoid complications in mothers as well as newborns. Currently, diet and insulin are important and the commonly used options in the treatment of GDM [4]. Insulin therapy is considered as the gold standard and treatment of choice in GDM [14,15]. It is advised to women not controlled on diet [16]. While insulin and lifestyle modifications are insufficient to provide effective relief, certain oral antihyperglycemic drugs can be considered as alternative treatment options [17]. In this study, we compared outcomes of GDM and newborns in mothers treated with insulin and acarbose.

In our study, all baseline characteristics including body mass index, comorbidities, family history of diabetes, history of GDM among patients, mean fasting glucose and postprandial blood glucose at the time of diagnosis of GDM in acarbose and insulin group were similar except mean age. Mean age in patients in insulin group was more than those in the acarbose group.

A small case series of six patients reported normalization of fasting and postprandial glucose levels in all cases. All pregnancies were uneventful and the newborns were normal [18]. Berini et al. compared neonatal results in 70 mothers with GDM treated with insulin, glyburide and acarbose [11]. There was no difference in the maternal characteristics between groups and glucose control was not achieved in higher percentage of patients with acarbose than glyburide. There was no difference in fasting and post-prandial glucose levels between the three groups [11]. In our study, there was no significant difference in the reduction of fasting and postprandial blood glucose level at day seven and after six weeks between insulin and acarbose groups. Similarly, mean HbA1c levels in insulin group and acarbose group at six weeks were similar.

In our study, there was no significant difference in the insulin and acarbose groups for the rates of recurrent infections, or preeclampsia. Similarly, the rates of premature rupture of membranes (PROM) were similar between two groups. A meta-analysis of oral antidiabetic drugs and insulin in GDM reported highest incidence of preeclampsia and hyperbilirubinemia with glyburide [19]. A Cochrane review reported increased risk of hypertensive disorders of pregnancy with insulin as compared to oral anti-diabetic drugs [20]. Metformin (plus insulin when required) is associated with low incidence of pregnancy induced hypertension 
[19]. A Cochrane analysis reported no difference between insulin and oral anti-diabetic drugs for the risk of preeclampsia [20]. According to the results of another meta-analysis, insulin had significantly higher risk of preeclampsia than metformin [21].

In our study, there was no significant difference in the modes of delivery between two groups. The mean duration of gestational week at the time of delivery in the insulin treated group and acarbose treated groups was similar. A Cochrane review also reported no difference between insulin and oral anti-diabetic drugs for the risk of birth by caesarean section [20]. In our study, there was no difference in the incidence of complications during delivery including birth trauma, shoulder dystocia, birth asphyxia or neonatal jaundice between patients treated with insulin versus acarbose respectively. However, birth trauma was mostly seen in instrumental vaginal delivery and some of the lower segment caesarean section cases. Injuries were mostly due to blades of forceps. In one case, there was a fractured humerus and the baby was delivered by assisted breech. Birth asphyxia was due to meconium aspiration and macrosomic babies leading to a prolonged second stage of labour.

We did not observe significant difference in the mean birth weight of babies born to mothers treated with insulin versus acarbose. A total of $4 \%$ and $8 \%$ babies in the insulin group and acarbose group had birth weight of more than $3500 \mathrm{~g}$ respectively. In another study, there was no difference in newborn weight in mothers with GDM treated with insulin, glyburide and acarbose [11]. Significant increase in birth weight and gestational age at delivery has been reported with insulin as compared to metformin [21]. In a meta-analysis of oral antidiabetic drugs and insulin, shortest gestational age at delivery and lowest mean birth weight has been reported with glyburide [19]. Metformin (plus insulin when required) is associated with low incidence of low birth weight and low gestational age at delivery [19].

Rate of macrosomia has been reported to be higher with glyburide than acarbose [11]. A meta-analysis of oral antidiabetic drugs and insulin in GDM reported glyburide to have highest incidence of macrosomia [19]. Metformin is associated with low incidence of macrosomia [19]. According to the results of a meta-analysis, insulin had significantly higher risk macrosomia than metformin [21].

In our study, there was no significant difference in the mean random blood glucose levels in babies born to mothers after insulin treatment versus those born to mothers treated with acarbose. Similarly, the rate of new-born babies with random blood glucose less than $45 \mathrm{mg} / \mathrm{dl}$ after delivery was similar in both groups. A meta-analysis of oral antidiabetic drugs and insulin in GDM reported highest incidence of neonatal hypoglycemia with glyburide [19]. In a systematic review, insulin was reported to have higher rates of neonatal hypoglycemia than metformin [22]. In another study, neonatal hypoglycemia was observed in more cases with glyburide than insulin and acarbose [11]. A meta-analysis of oral antidiabetic agents like insulin reported lowest risk of neonatal hypoglycemia with acarbose [19]. Neonatal hypoglycemia is one of the important concerns with insulin in gestational diabetes. A study reported 33\% and $35 \%$ incidence of mild and severe hypoglycemia with insulin respectively [23]. However, in a Cochrane review, there was no evidence of a clear difference between insulin and oral antidiabetic therapy [20]. In another meta-analysis, glyburide had a higher increase of neonatal hypoglycemia compared to insulin [21]. According to the results of a meta-analysis, insulin had significantly higher risk of neonatal hypoglycemia than metformin [21].

In a systematic review, rate of congenital malformations was similar with insulin and oral drugs [22]. In our study, congenital anomaly was seen only in one case of the insulin treated group in form of congenital talipes equinovarus. No cases of congenital anomaly were seen in acarbose treated group.

In a study amongst metformin, glyburide and insulin, insulin had the highest incidence of neonatal intensive care unit admission [19]. Metformin has been reported to be associated with low incidence of respiratory distress syndrome [19]. According to the results of a meta-analysis, insulin had significantly higher risk of neonatal intensive care unit admission than metformin [21]. In our study, there was no difference in admission to special new born care unit between patients treated with insulin versus acarbose. The causes of admission to special new born care unit were mostly due to neonatal jaundice, birth asphyxia, hypoglycaemia, preterm delivery and intrauterine growth retardation. All cases of neonatal jaundice had unconjugated hyperbilirubinemia which included both physiological and pathological jaundice.

Acarbose is associated with intestinal discomfort [18]. In our study, acarbose was well tolerated by the patients without any major side effects. Overall, our study suggests promising role of acarbose in patients with GDM. The results of an on-going multicentre study ACARB-GDM will provide more insights on the outcomes in patients with GDM [13].

Our study has some limitations. It was conducted at a single center and the relatively small sample size are the other limitations. Larger, multicentre studies are required to confirm our observations.

\section{Conclusions}

In our study, there were no significantly adverse neonatal or maternal outcomes or congenital anomalies in the acarbose treated group of patients with GDM. The glycemic control in patients with GDM was 
comparable between groups treated with acarbose and insulin. Our preliminary results suggest that acarbose is an attractive option for treatment of GDM. More studies involving large number of patients are required for the recommendation of use of acarbose in GDM.

\section{Additional Information \\ Disclosures}

Human subjects: Consent was obtained by all participants in this study. IEC/IRB issued approval IEC/IRB No. 504. The study was approved by the Institutional Ethics Committee. Animal subjects: All authors have confirmed that this study did not involve animal subjects or tissue. Conflicts of interest: In compliance with the ICMJE uniform disclosure form, all authors declare the following: Payment/services info: All authors have declared that no financial support was received from any organization for the submitted work. Financial relationships: All authors have declared that they have no financial relationships at present or within the previous three years with any organizations that might have an interest in the submitted work. Other relationships: All authors have declared that there are no other relationships or activities that could appear to have influenced the submitted work.

\section{References}

1. Moon JH, Kwak SH, Jang HC: Prevention of type 2 diabetes mellitus in women with previous gestational diabetes mellitus. Korean J Intern Med. 2017, 32:26-41. 10.3904/kjim.2016.203

2. Hunt KJ, Schuller KL: The increasing prevalence of diabetes in pregnancy . Obstet Gynecol Clin North Am. 2007, 34:173-10. 10.1016/j.ogc.2007.03.002

3. Plows JF, Reynolds CM, Vickers MH, Baker PN, Stanley JL: Nutritional supplementation for the prevention and/or treatment of gestational diabetes mellitus. Curr Diab Rep. 2019, 18:73. 10.1007/s11892-019-1199-1

4. Brzozowska M, Bieniek E, Szosland K, Lewinski A: Gestational diabetes - is diet and insulin the only solution?. Neuro Endocrionol Lett. 2017, 38:311-15.

5. Eades CE, Cameron DM, Evans JMM: Prevalence of gestational diabetes mellitus in Europe: a meta-analysis . Diabetes Res Clin Pract. 2017, 129:173-81. 10.1016/j.diabres.2017.03.030

6. Agha-Jaffar R, Oliver N, Johhston D, Robinson S: Gestational diabetes mellitus: does an effective prevention strategy exist?. Nat Rev Endocrinol. 2016, 12:533-46. 10.1038/nrendo.2016.88

7. Krishnaveni GV, Hill JC, Veena SR, et al.: Gestational diabetes and the incidence of diabetes in the 5 years following the index pregnancy in South Indian women. Diabetes Res Clin Pract. 2007, 78:398-404. 10.1016/j.diabres.2007.06.002

8. Szmuilowicz ED, Josefson JL, Metzger BE: Gestational diabetes mellitus. Endocrinol Metab Clin North Am. 2019, 48:479-493. 10.1016/j.ecl.2019.05.001

9. Johns EC, Denison FC, Norman JE, Reynolds RM: Gestational diabetes mellitus: mechanisms, treatment, and complications. Trends Endocrinol Metab. 2018, 29:743-54. 10.1016/j.tem.2018.09.004

10. Kalra B, Gupta Y, Singla R, Kalra S: Use of oral anti-diabetic agents in pregnancy: a pragmatic approach . N Am J Med Sci. 2015, 7:6-12. 10.4103/1947-2714.150081

11. Berini AM, Silva JC, Taborda W, et al.: Perinatal outcomes and the use of oral hypoglycemic agents . J Perinat Med. 2005, 33:519-23. 10.1515/JPM.2005.092

12. Balsells M, Garcia-Patterson A, Sola I, Rogue M, Gich I, Corcoy R: Glibenclamide, metformin, and insulin for the treatment of gestational diabetes: a systematic review and meta-analysis. BMJ. 2015:350, 102:10.1136/bmj.h102

13. Acarbose and prandial insulin for the treatment of gestational diabetes mellitus . (2017). Accessed: December 25, 2020: https://clinicaltrials.gov/ct2/show/NCT03380546.

14. Corcoy R, Balsells M, Garcia-Patterson A, Shmueli A, Hadar E: Pharmacotherapy for hyperglycemia in pregnancy-do oral agents have a place?. Diabetes Res Clin Pract. 2018, 145:51-58. 10.1016/j.diabres.2018.04.015

15. Nicholson W, Baptiste-Roberts K: Oral hypoglycaemic agents during pregnancy: the evidence for effectiveness and safety. Best Pract Res Clin Obstet Gynaecol. 2011, 25:51-63. 10.1016/j.bpobgyn.2010.10.018

16. Subiabre M, Silva L, Toledo F, et al.: Insulin therapy and its consequences for the mother, foetus, and newborn in gestational diabetes mellitus. Biochim Biophys Acta Mol Basis Dis. 2018, 1864:2949-2956. 10.1016/j.bbadis.2018.06.005

17. Mukerji G, Feig DS: Pharmacological management of gestational diabetes mellitus . Drugs. 2017, 77:1723-32. 10.1007/s40265-017-0807-0

18. Zarate A, Ochoa R, Hernandez M, Basurto L: Effectiveness of acarbose in the control of glucose tolerance worsening in pregnancy. Ginecol Obstet Mex. 2000, 68:42-5.

19. Liang H-L, Ma S-J, Xiao Y-N, Tan H-Z: Comparative efficacy and safety of oral antidiabetic drugs and insulin in treating gestational diabetes mellitus: an updated PRISMA-compliant network meta-analysis. Medicine (Baltimore). 2017, 96:7939. 10.1097/MD.0000000000007939

20. Brown J, Grzeskowiak L, Williamson K, Downie MR, Crowther CA: Insulin for the treatment of women with gestational diabetes. Cochrane Database Syst Rev. 2017, 11:CD012037. 10.1002/14651858.CD012037.pub2

21. Guo L, Ma J, Tang J, Hu D, Zhang W, Zhao X: Comparative efficacy and safety of metformin, glyburide, and insulin in treating gestational diabetes mellitus: a meta-analysis. J Diabetes Res. 2019, 2019:9804708. $10.1155 / 2019 / 9804708$

22. Nicholson W, Bolen S, Witkop CT, Neale D, Wilson L, Bass E: Benefits and risks of oral diabetes agents compared with insulin in women with gestational diabetes: a systematic review. Obstet Gynecol. 2009, 113:193-205. 10.1097/AOG.0b013e318190a459

23. Voormolen DN, de Wit L, van Rijn BB, et al.: Neonatal hypoglycemia following diet-controlled and insulin- 


\section{Cureus}

treated gestational diabetes mellitus. Diabetes Care. 2018, 41:1385-1390. 10.2337/dc18-0048 